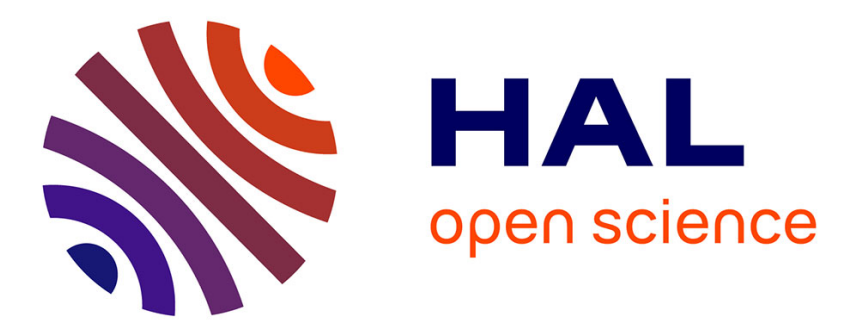

\title{
A new optimized Stockwell transform applied on synthetic and real non-stationary signals
}

Ali Moukadem, Bouguila Zied, Djaffar Ould-Abdeslamb, Alain Dieterlen

\section{To cite this version:}

Ali Moukadem, Bouguila Zied, Djaffar Ould-Abdeslamb, Alain Dieterlen. A new optimized Stockwell transform applied on synthetic and real non-stationary signals. Digital Signal Processing, 2015, 46, pp.226-238. 10.1016/j.dsp.2015.07.003 . hal-01291611

\section{HAL Id: hal-01291611 \\ https://hal.science/hal-01291611}

Submitted on 20 Jan 2022

HAL is a multi-disciplinary open access archive for the deposit and dissemination of scientific research documents, whether they are published or not. The documents may come from teaching and research institutions in France or abroad, or from public or private research centers.
L'archive ouverte pluridisciplinaire HAL, est destinée au dépôt et à la diffusion de documents scientifiques de niveau recherche, publiés ou non, émanant des établissements d'enseignement et de recherche français ou étrangers, des laboratoires publics ou privés.

\section{다)(1) $(5$}

Distributed under a Creative Commons Attribution - NonCommerciall 4.0 International 


\title{
A new optimized Stockwell transform applied on synthetic and real non-stationary signals
}

\author{
Ali Moukadem*, Zied Bouguila, Djaffar Ould Abdeslam, Alain Dieterlen \\ University of Haute Alsace, MIPS Laboratory, Mulhouse, France
}

The aim of this paper is to improve the energy concentration of the Stockwell transform (S-transform) in the time-frequency domain. Amodified S-transform is proposed with several parameters to control the width of a hybrid Gaussian window. Aconstrained optimization problem is proposed based on an energy concentration measure as objective function and inequalities constraints to define the bounds of the Gaussian window. An active-set algorithm is applied to resolve the optimization problem. The optimization of the energy concentration in the time-frequency plane can lead to more reliable applications for non-stationary signals. The simulation results show a significant improvement of the proposed methodology most notably in the presence of noise comparing with the standard S-transform and existing modified Stransform in the literature. Moreover, comparison with other known time-frequency transforms such as Short-time Fourier transform (STFT) and smoothed-pseudo Wigner-Ville distribution (SPWVD) is also performed and discussed. The proposed S-transform is tested also on real non-stationary signals through an example of split detection in heart sounds.

\section{Introduction}

Time-Frequency analysis is a powerful tool to describe signals both in the time and in the frequency domain. It transforms a one dimensional signal $x(t)$ into a two-dimensional function of time and frequency $T_{X}(t, f)[1]$. This can be done by several approaches. The first class of solutions is named the linear time-frequency representations methods which the well-known Short-Time Fourier Transform (STFT) and the Wavelet-Transform (WT) are part of the main concept used in those approaches lies in the signal decomposition into elementary parts (atoms) and tries to localize each part in time and frequency properly and simultaneously. The second approach concerning the Time-Frequency (TF) methods is the quadratic transforms which aim at distributing the energy of the signal over the two description variables: time and frequency. Each approach has some advantages and drawbacks; while linear timefrequency representations are intuitive they suffer from poor $\mathrm{TF}$ resolution in many cases. This depends on the windows used to analyze the signal. On the other hand, the quadratic transforms (the Wigner-Ville for example) have a high TF resolution. However, they suffer from cross-terms in multicomponent signals and may also suffer from inner interference for non-linear mono-component

\footnotetext{
* Corresponding author.

E-mail address: ali.moukadem@uha.fr (A. Moukadem).
}

signal. There is no time-frequency method which can be considered as optimal for all applications.

The Stockwell transform (S-transform) can be considered as a hybrid between the Short Time Frequency Transform (STFT) and the wavelet transform [2]. It can be viewed as a frequency dependent STFT or a phase corrected wavelet transform. It has gained popularity in the signal processing community because of its easy interpretation and fast computation [3]. The S-transform has been shown high performance in classification and feature extraction problems applied on non-stationary signals, such as heart sounds [4-6], power quality signals [7], EEG signals [8] etc. Generally the S-transform uses a Gaussian window, whose standard deviation varies over frequency. Whatever the analyzed signal, the width of the Gaussian window will decrease as the frequency increases. This produces a higher frequency resolution at lower frequencies and a higher time resolution at higher frequencies. This can be considered as limitation in some signal analysis, for example, for a signal containing a single sinusoid, the time-frequency localization can be considerably improved if the window is very narrow in the frequency domain. Similarly, for signals containing only a Dirac impulse, it would be beneficial for good time-frequency localization to have very wide window in the frequency domain [9]. It would be more appropriate to adapt the window to the signal in order to maximize the energy localization of the S-transform.

Many studies in the literature tried to improve the Stockwell transform by proposing new windows. McFadden et al. [10] pro- 
posed a generalized S-transform which provides greater control on the window function. Later, Pinnegar et al. [11] proposed another generalized S-transform in which two prescribed functions of frequency control the scale and the shape of the analyzing window. The same authors proposed another modified S-transform with a bi-Gaussian window which seems better at resolving the sharp onset of events in a time series [12]. Sejdic et al. and Djurovic et al. $[9,13]$ introduced a novel parameter to the Gaussian window and the parameter which maximizes the concentration energy is selected [9]. This is the main study in the literature interested to optimize the energy concentration directly in the TF domain, that is, to minimize the spread of the energy beyond the actual signal components. More recently, Assous et al. [14] proposed another modified S-transform in which the scaling parameter of the Gaussian window varies linearly with the frequency.

The energy concentration in the Time-Frequency (TF) domain is a very important criteria for the algorithms that aim to detect or extract relevant feature from time-frequency domain. Hence, the importance of an energy concentration optimization process to improve the detection and the classification of non-stationary signals. As it is well known, the ideal time-frequency transformation should only be distributed along frequencies for the duration of signal components. So the neighboring frequencies would not contain any energy and the energy contribution of each component would not exceed its duration [15]. In this paper, we adopt the strategy proposed by Sejdic et al. [9], that is, to adapt the analyzed window to the energy concentration criteria.

The main contributions of this paper can be summarized as:

- Proposing a methodology to optimize the energy concentration of the S-transform. For that, new parameters are introduced to control better the width of the Gaussian window and an active-set algorithm is applied to select properly these parameters.

- An application of the modified S-transform on the detection of splits in heart sounds is proposed.

This paper is an improved and extended version of the paper published in [16]. The paper is organized as follows: Section 2 presents the proposed modified S-transform with the optimization problem. Section 3 presents the simulation study to compare the proposed method to other existing S-transform where the robustness against noise and the performance of estimation of instantaneous frequency are discussed and detailed. A comparison with other classic time-frequency representations is also discussed. Section 4 tries to show the importance of the time-frequency resolution enhancement in the detection of real non-stationary signals by presenting an application on heart sounds. Finally, Section 5 gives the conclusion and the future work.

\section{Optimization of the modified Stockwell transform}

\subsection{The original S-transform and the link with Fourier}

The original S-transform of a time varying signal $x(t)$ is defined by $[2]$ :

$S_{x}(\tau, f)=\int_{-\infty}^{+\infty} x(t) w(t-\tau, f) e^{-2 \pi j f t} d t$

where the window function $w(t, f)$ is chosen as:

$w(t, f)=\frac{1}{\sigma(f) \sqrt{2 \pi}} e^{\frac{-t^{2}}{2 \sigma(f)^{2}}}$

and $\sigma(f)$ is a function of frequency as: $\sigma(f)=\frac{1}{|f|}$

The window is normalized as:

$\int_{-\infty}^{+\infty} w(t, f) d t=1$

This gives the direct relation between the S-transform and the Fourier spectrum by averaging the local spectrum over time:

$\int_{-\infty}^{+\infty} S_{X}(\tau, f) d t=X(f)$

where $X(f)$ is the Fourier transform of $x(t)$. The signal $x(t)$ can be recovered from $S_{\tau}(t, f)$ as follows:

$x(t)=\int_{-\infty}^{+\infty}\left\{\int_{-\infty}^{+\infty} S_{x}(\tau, f)\right\} e^{i 2 \pi f t} d f d \tau$

Another way to directly express the link between the S-transform and the Fourier transform is by writing the ST as a convolutions process as follows:

$$
\begin{aligned}
S_{X}(\tau, f) & =\int_{-\infty}^{+\infty} p(t, f) g(\tau-t, f) d t \\
& =p(\tau, f) * g(\tau, f)
\end{aligned}
$$

where:

$p(\tau, f)=x(\tau) e^{-i 2 \pi f \tau}$

and:

$g(\tau, f)=\frac{|f|}{\sqrt{2 \pi}} e^{\frac{-\tau^{2} f^{2}}{2}}$

By calculating the Fourier transform of $S_{X}(\tau, f)$, the convolution becomes a multiplication in the frequency domain:

$$
\begin{aligned}
F_{\tau \rightarrow \alpha}\left\{S_{X}(\tau, f)\right\} & =P(\alpha, f) G(\alpha, f) \\
& =X(\alpha+f) e^{\frac{-2 \pi^{2} \alpha^{2}}{f^{2}}}
\end{aligned}
$$

where $P(\alpha, f)$ and $G(\alpha, f)$ are as the corresponding Fourier transforms for $p(\tau, f)$ and $g(\tau, f)$, respectively and $\alpha$ is the frequency Fourier variable related to $\tau$. The direct relation between the S-transform and the Fourier transform can be obtained by applying the inverse Fourier transform to the last equation:

$S(\tau, f)=\int_{-\infty}^{+\infty} X(\alpha+f) e^{\frac{-2 \pi^{2} \alpha^{2}}{f^{2}}} e^{i 2 \pi \alpha \tau} d \alpha$

This will facilitate the implementation of the ST by using the advantages of the FFT (Fast Fourier Transform) algorithms. The exponential function in Eq. (10) is the frequency dependent localizing window. This window is centered on the zero frequency and thus plays the role of a low pass filter for each particular voice.

\subsection{Energy concentration enhancement}

It has been shown that the original S-transform uses a Gaussian window, whose standard deviation varies over frequency. Whatever the analyzed signal, the width of the Gaussian window will decrease as the frequency increases. As we mentioned it above, this strategy can be considered as a limitation since it does not 
take the nature of the analyzed signal into consideration. It would be more appropriate to adapt the window to the signal in order to maximize the resolution of the S-transform. We will present the main modified S-transform existing in the literature before presenting the proposed modified S-transform in this paper with the optimization methodology.

\subsubsection{The modified S-transforms in the literature}

The first modification made on the classic Gaussian window of the S-transform was to introduce a novel parameter to better control the resolution [17]. The parameter $\gamma$ is introduced as follows:

$w(\tau-t, f, \gamma)=\frac{|f|}{\gamma \sqrt{2 \pi}} e^{\frac{-f^{2}(\tau-t)^{2}}{2 \gamma^{2}}}$

This allows a better control of the time-frequency resolution of the S-transform by controlling the width of the Gaussian window. Another way to control the width of Gaussian window in the S-transform is proposed by Sejdic et al. [9] and consists to fix $\gamma$ to 1 and introduce a new parameter $p$ as follows:

$w(\tau-t, f, p)=\frac{|f|^{p}}{\sqrt{2 \pi}} e^{\frac{-f^{2 p}(\tau-t)^{2}}{2}}$

The equation (13) can be rewritten as:

$w(\tau-t, f, p)=\frac{|f|}{f^{(1-p)} \sqrt{2 \pi}} e^{\frac{-f^{2}(\tau-t)^{2}}{2 f^{(1-p)^{2}}}}$

In this case $\gamma$ can be written as follows:

$\gamma(f)=f^{(1-p)}$

Recently, George et al. [18] and Assous et al. [14] proposed another way to control the Gaussian window by varying the scaling parameter $\gamma$ linearly with frequency as follows:

$\gamma(f)=m f+k$

This modification provides a better progressive control of the window width.

\subsubsection{The proposed window}

In this paper, we propose to generalize the modifications proposed by [9] and $[18,14]$ by introducing a new Gaussian window with the following standard deviation:

$\sigma(f)=\frac{m f^{p}+k}{f^{r}}$

In this case the modified Gaussian window can be given as:

$w(\tau-t, f)=\frac{|f|^{r}}{\left(m f^{p}+k\right) \sqrt{2 \pi}} e^{\frac{-(\tau-t)^{2} f^{2 r}}{2\left(m f^{p}+k\right)^{2}}}$

The parameter $f^{r} /\left(m f^{p}+k\right)$ represents the number of cycles (periods) of a frequency that can be contained within one standard deviation of the Gaussian window.

The introduced parameters $m, p, k$ and $r$ aim to give more flexibility to the Gaussian window. The modified S-transform becomes:

$S_{x}^{m, p, k, r}(\tau, f)=\int_{-\infty}^{+\infty} x(t) \frac{|f|^{r}}{\left(m f^{p}+k\right) \sqrt{2 \pi}} e^{\frac{-(\tau-t)^{2} f^{2 r}}{2\left(m f^{p}+k\right)^{2}}} e^{-i 2 \pi f t} d t$

The new window satisfies the normalization condition for the original S-transform window which insures the invertibility of the modified S-transform:

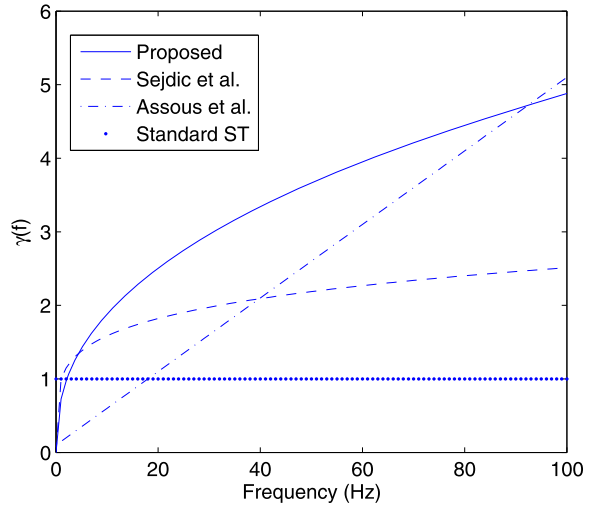

Fig. 1. The variation of function $\gamma$ over frequency for: proposed ST ( $m=0.3$, $p=0.0386, k=0.4276, r=0.6035)$; (dashed line) Sejdic's ST ( $m=0, p=1, k=1$ $r=0.8$ ); (dashed dotted line) Assous's ST ( $m=0.05, p=1, k=0.1, r=1)$; (dotted line) standard ST ( $m=1, p=0, k=0, r=1)$.

$\int_{-\infty}^{+\infty} \frac{|f|^{r}}{\left(m f^{p}+k\right) \sqrt{2 \pi}} e^{\frac{-(\tau-t)^{2} f^{2 r}}{2\left(m f^{p}+k\right)^{2}}} d t=1$

As in equation (14) the windows in (18) can be rewritten as:

$w(\tau-t, f)=\frac{|f|}{\left(m f^{p}+k\right) f^{(1-r) \sqrt{2 \pi}}} e^{\frac{-(\tau-t)^{2} f^{2}}{2\left(\left(m f^{p}+k\right) f^{(1-r)}\right)^{2}}}$

and then the scaling parameter $\gamma(f)$ function can be given as:

$$
\begin{aligned}
\gamma(f) & =\left(m f^{p}+k\right) f^{(1-r)} \\
& =m f^{(p-r+1)}+k f^{(1-r)}
\end{aligned}
$$

The scaling rule for the proposed Gaussian window is made as a polynomial function of frequency. The introduced parameters aims to make the Gaussian window more flexible and more adaptive to the analyzed signal. We note here that we can retrieve Sejdic's window from equation (21) by setting $m=0$ and $k=1$ and Assous's window by setting $p=1$ and $r=1$ and the standard ST by setting $m=0, k=1$ and $r=0$. Fig. 1 shows examples of the variation of $\gamma$ function over frequency for the different windows.

\subsubsection{Optimization methodology to select optimal parameters}

A crucial question is how to choose the parameters of the Gaussian window? Assous et al. select empirically the value of $m$ and $k$, $k$ is fixed to $1 / N$ where $N$ is the number of signal's samples and $m$ is fixed to four times the variance of the signal [14]. Select empirically the values of parameters may not be adequate for some types of signals. It will be more appropriate to generate automatically adaptive parameters which respect the nature of analyzed signal. The modified S-transform proposed by Sejdic et al. [9] introduce one parameter to the Gaussian window and this parameter is varied iteratively over the selected interval and the value which maximize the concentration energy is selected. In this paper, since we have several parameters that control the Gaussian window, we propose to apply a more advanced optimization methodology to select automatically $m, p, k$ and $r$.

The objective function used in this paper is the energy concentration measure (CM) proposed in [19]. By applying this measure to the modified S-transform, we obtain:

$$
C M(m, p, k, r)=\frac{1}{\int_{-\infty}^{+\infty} \int_{-\infty}^{+\infty}\left|\overline{S_{x}^{m, p, k, r}(t, f)}\right| d t d f}
$$

where the module of the S-transform is normalized as 


$$
\overline{S_{x}^{m, p, k, r}(t, f)}=\frac{S_{x}^{m, p, k, r}(t, f)}{\sqrt{\int_{-\infty}^{+\infty} \int_{-\infty}^{+\infty}\left|S_{x}^{m, p, k, r}(t, f)\right|^{2} d t d f}}
$$

Then, the optimization problem can be expressed as follows:

$\underset{m, p, k, r}{\arg \max }\left(1 / \int_{-\infty}^{+\infty} \int_{-\infty}^{+\infty}\left|\overline{S_{x}^{m, p, k, r}(t, f)}\right| d t d f\right)$

The constraints of our optimization problem are related to the width bounds of the analyzed window. The window should not be very narrow which alters the time resolution and not very large which destroys the frequency resolution

$L_{b}<\sigma(f)<U_{b}$

with $L_{b}$ being chosen as $n T_{s}$ and $U_{b}$ as $l T_{s}$ where $T_{s}$ is a sampling period of the discretized window. Theoretically, the integer $n$ should be greater than 2 , in this paper $n$ is fixed empirically as 10 and $l$ is fixed as 1000 , so for a sampling frequency of $1000 \mathrm{~Hz}$ $\left(T_{s}=0.001 \mathrm{~s}\right) U_{b}$ is about 1 second.

By taking the first inequality of equation (26) we obtain:

$\frac{m f^{p}+k}{f^{r}} \geq n T_{s}$

We have $f \in\left[f_{\min }, f_{\max }\right] . f_{\min }$ is fixed to $1 \mathrm{~Hz}$ however $f_{\max }$ depends on the analyzed signal. By developing equation (27) and by majorating the two sides we obtain:

$m f_{\min }^{p}+k \geq n T_{s} f_{\max }^{r}$

Since $f_{\min }$ is generally set to $1 \mathrm{~Hz}$, this allows us to write the first constraint as follows:

$n T_{s} f_{\text {max }}^{r}-k-m \leq 0$

By following the same methodology, the second constraint can be written as:

$m f_{\text {max }}^{p}+k-l T_{s} \leq 0$

The third constraint delimits the interval of the parameters $m, p, k$ and $r$ which is chosen between [0,3]. The lower bound is set to 0 to ensure to have a positive windows width and the upper bound 3 is chosen based on our experience. Then the overall optimization problem can be written as:

$\underset{m, p, k, r \in \mathbb{R}}{\arg \max }\left(1 / \sum_{1}^{N} \sum_{f_{\min }}^{f_{\max }}\left|\overline{S_{x}^{m, p, k, r}(t, f)}\right| d t d f\right)$

Subject to: $n T_{s} f_{\max }^{r}-k-m \leq 0$

$$
m f_{\max }^{p}+k-l T_{s} \leq 0
$$$$
0 \leq m, p, k, r \leq 3
$$

This can be considered as nonlinear optimization problem with constraints. An active-set strategy is applied. The principle behind active-set algorithms states that if a minimizer on each working surface is found during each iteration within the defined active-set region and there is a decrease in the value of the objective function at each iteration then the algorithm terminates after finitely many iterations. So if there is a solution that satisfies the constraints within the given feasible region then the algorithm terminates. In [16] we have used Genetic Algorithm (GA) to resolve the optimization problem without constraints. However, by adding the respective constraints to ensure the width limits of the Gaussian window, the GA became very time consuming. The active-set was used as it requires the least computation time and it seems to give a very satisfied results as we will show in the results later.

\section{Illustration and comparison on test synthetic signals}

Firstly, we apply the proposed S-transform on synthetic signals and we compare its energy concentration with two main groups of time-frequency representations:

- The standard Stockwell transform and the main modified versions proposed in the literature by Sejdic et al. and Assous et al. $[9,14]$.

- The Short-time Fourier Transform (STFT) and the smoothedpseudo Wigner-Ville distribution (SPWVD).

We propose to compare with three classes of synthetic signals: signals with sinusoidal modulated components and crossing linear chirp components $x_{1}(t)$, signals with four short duration transients $x_{2}(t)$ and signals with fast frequency variation and crossing components $x_{3}(t)$.

The synthetic signals can be given as:

$x_{1}(t)=\cos \left(a \pi t-b \pi t^{2}\right)+\cos (4 \pi \sin (f \pi t)+80 \pi t)$

where the introduced parameters ( $a, b$ and $f$ ) control the chirp rate and the frequency of the sinusoidal modulated component and they are chosen as follows: $80 \leq a \leq 200,20 \leq b \leq 80$ and $1 \leq f \leq 10$

$$
\begin{aligned}
x_{2}(t)= & e^{-35 \pi\left(t-t_{1}\right)^{2}} \cos \left(f_{1} \pi t\right)+e^{-35 \pi\left(t-t_{2}\right)^{2}} \cos \left(f_{1} \pi t\right) \\
& +e^{-55 \pi\left(t-t_{3}\right)^{2}} \cos \left(f_{2} \pi t\right)+e^{-45 \pi\left(t-t_{3}\right)^{2}} \cos \left(f_{3} \pi t\right)
\end{aligned}
$$

where the introduced parameters $\left(t_{1}, t_{2}, t_{3}, f_{1}, f_{2}\right.$ and $\left.f_{3}\right)$ control the time and frequency positions of the Gaussian modulated kernels and they are chosen as follows: $t_{2}=t_{1}+\alpha, t_{3}=t_{1}+\left(t_{2}-t_{1}\right) / 2$ with $0.1 \leq t_{1} \leq 0.3$ and $0.2 \leq \alpha \leq 0.5 f_{2}=f_{1}+\beta, f_{3}=f_{1}-\beta$ with $20 \leq f_{1} \leq 70$ and $15 \leq \beta \leq 40$

$x_{3}(t)=\cos (20 \pi \ln (a t+1))+\cos \left(b \pi t+c \pi t^{2}\right)$

where the introduced parameters $(a, b$ and $c$ ) control the chirp rates of the two crossing components and they are chosen as follows: $1 \leq a \leq 50,1 \leq b \leq 100$ and $1 \leq c \leq 200$.

The introduced parameters in each signal are generated randomly in the adequate intervals in order to generate 100 realizations of each class of signals $\left(x_{1}, x_{2}, x_{3}\right)$.

\subsection{Comparison between different versions of Stockwell transform}

As it is mentioned above, Assous et al. select empirically the value of $m$ and $k$ [14]. Fig. 2 shows the limitation of this approach compared the proposed one. The synthetic signal used in the paper of Assous et al. is illustrated in Fig. 2 in order to show clearly that the optimization phase is very helpful to adapt the Gaussian parameters to the analyzed signal, hence to increase the time-frequency resolution $(\mathrm{CM}=0.0051$ and $\mathrm{CM}=0.0035$, respectively). We note here that in Fig. 2 the time resolution of the proposed method gives a little less time resolution than the Assous et al. method but much better frequency resolution. In the rest of this comparison section, and to be fare in the comparison, we will apply the same optimization methodology proposed in this paper to Assous's ST method and this will be named "optimized Assous's ST" method.

For the signal $x_{1}(t)(a=100, b=20$ and $f=5)$ (Fig. 3) the proposed approach gives clearly the better time-frequency representation. While the sinusoidal modulated component suffers from poor concentration in the standard ST overall the frequency band, Sejdic's ST method gives a poor resolution for this component for low frequencies as for the optimized Assous's ST. 
(a)

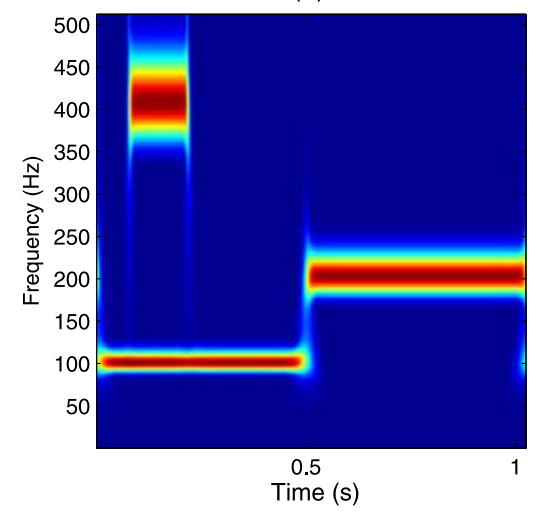

(b)

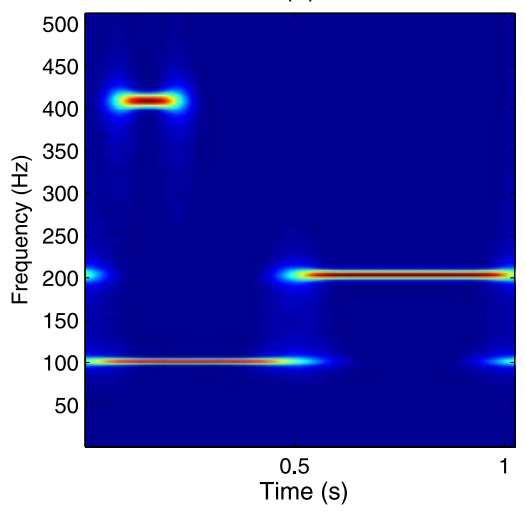

Fig. 2. Comparison between on the synthetic signal used in [14]: (a) Assous's ST [14]; (b) Proposed ST.

(a)

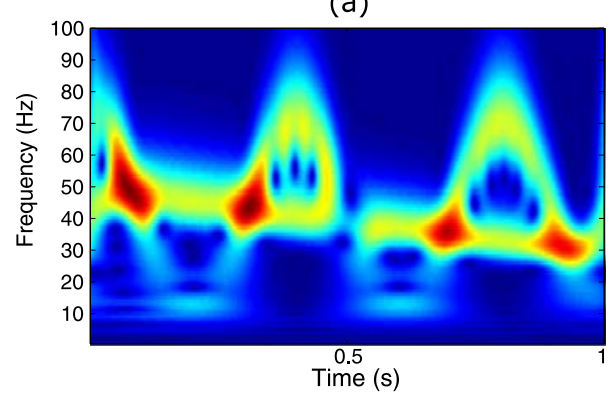

(c)

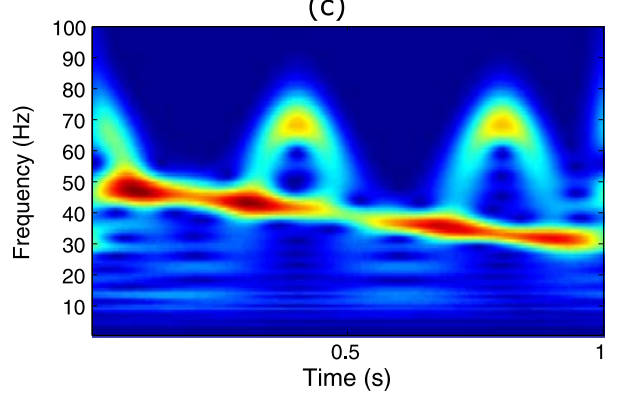

(b)

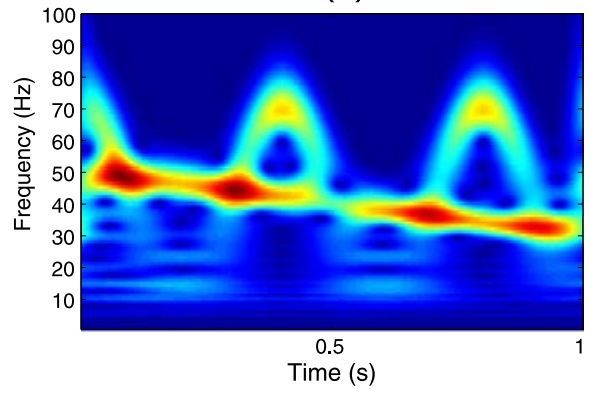

(d)

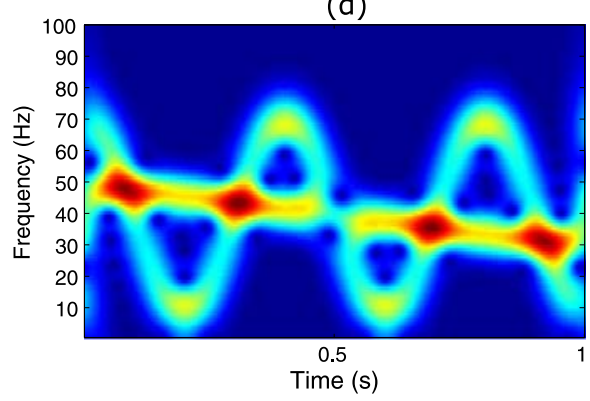

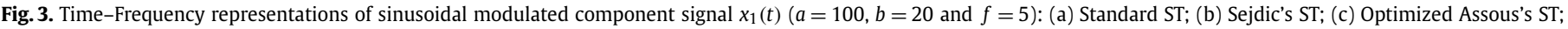
(d) The proposed ST.

Fig. 4 shows a four duration transients signal example for $x_{2}(t)$ $\left(t_{1}=0.3, t_{2}=0.7, t_{3}=0.5, f_{1}=45, f_{2}=75, f_{3}=15\right)$, where the standard ST suffers from bad frequency resolution in middle and high frequencies and bad temporal resolution in low frequencies. Sejdic's ST and optimized Assous's ST methods suffer also from bad temporal resolution for low frequencies. The proposed ST has a good time-frequency resolution overall the time-frequency plane.

For the cross-component signal example (Fig. 5) ( $a=30, b=40$ and $c=150$ ) the standard ST has a good resolution for the hyperbolic component since it follows the philosophy of the standard Gaussian window of the ST: a higher frequency resolution at lower frequencies and higher time resolution at higher frequencies. However, the resolution is decreased for the linear chirp component since the standard ST gives a poor frequency resolution for high frequencies. Sejdic's ST method has a good resolution for high and middle frequencies but a poor resolution for low frequencies as in Fig. 4. The optimized Assous's ST gives a better resolution for the hyperbolic component than Sejdic's ST but a worse resolution for the linear chirp especially for the high frequencies. The proposed ST gives a good resolution overall the time-frequency plane. This
Table 1

Shows the mean of the concentration energy measures (CM) calculated on 100 realizations of randomly generated parameters for each signal class and for different versions of ST.

\begin{tabular}{lllll}
\hline CM & Standard ST & Sejdic's ST & Optimized Assous's ST & Proposed ST \\
\hline$x_{1}(t)$ & 0.0043 & 0.0045 & 0.0047 & 0.0050 \\
$x_{2}(t)$ & 0.0083 & 0.0086 & 0.0086 & 0.0092 \\
$x_{3}(t)$ & 0.0047 & 0.0051 & 0.0052 & 0.0056 \\
\hline
\end{tabular}

example is used as demonstration to show that the proposed ST can deal with the problem of linear chirps. The generation of optimal parameters followed by the modified Gaussian window gives the proposed methodology the flexibility and the adaptability on the analyzed signal overall time-frequency plane. The concentration energy measures are calculated for 100 different parameters for each signal class and the different versions of ST are resumed in Table 1. The Kruskal-Wallis test is also applied on the obtained results of each method. A significant statistical differences are confirmed with a p-value $<0.05$. Fig. 6 illustrates the box plot for overall concentration energy measures and for each class of signals $\left(x_{1}, x_{1}\right.$ and $\left.x_{3}\right)$. 
(b)

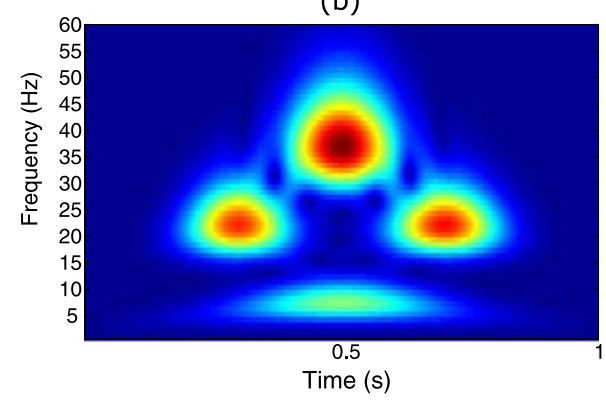

(d)

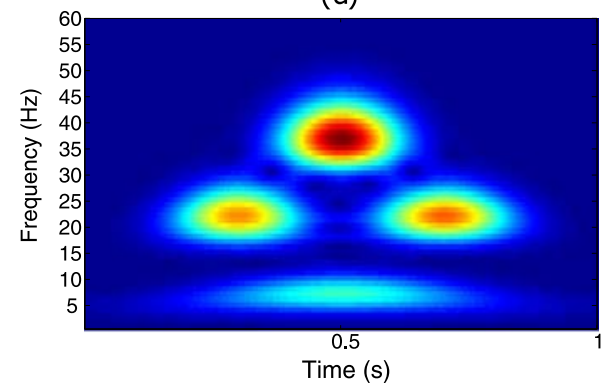

(c)

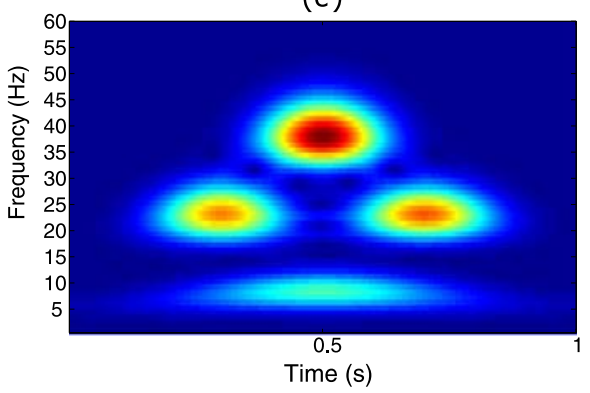

(a)

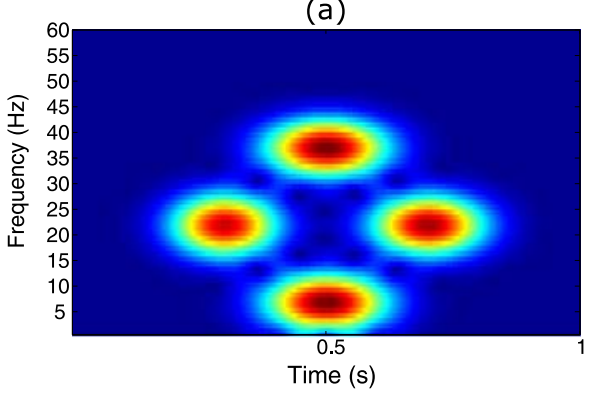

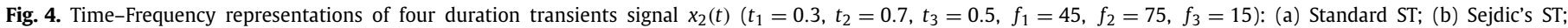
(c) Optimized Assous's ST; (d) The proposed ST.

(a)

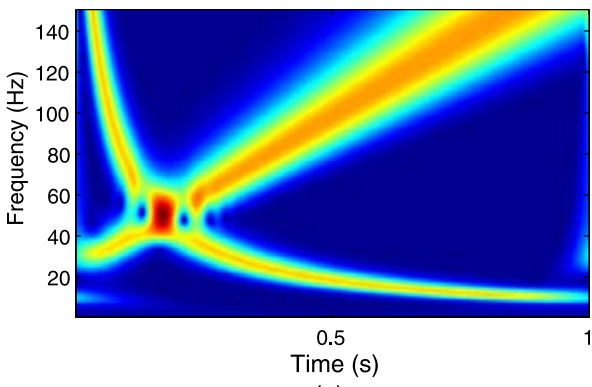

(c)

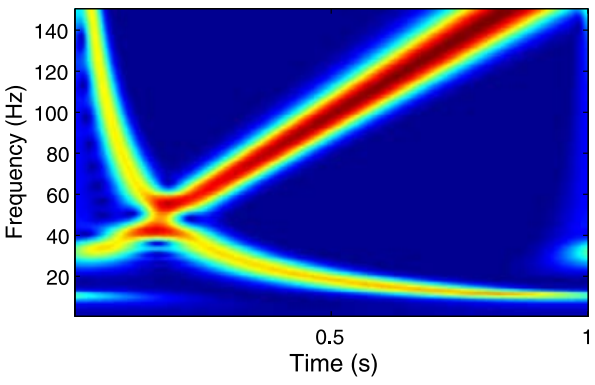

(b)

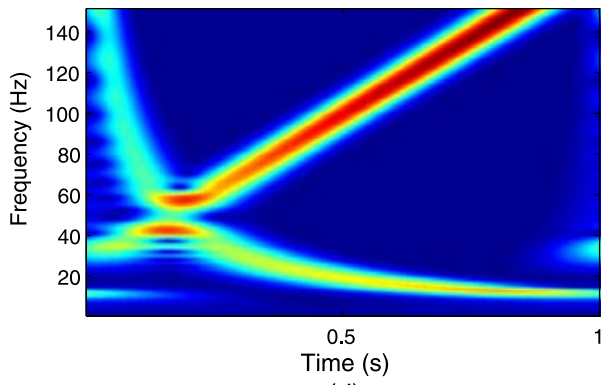

(d)

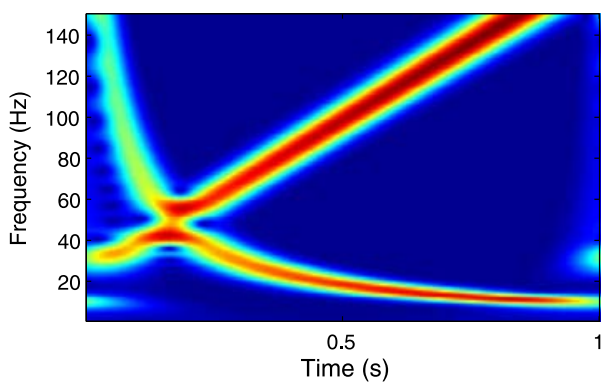

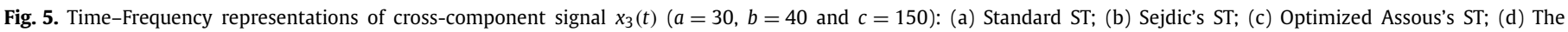
proposed ST.

\subsubsection{Robustness against noise}

To test the robustness of the proposed method on noisy signals, two levels of Gaussian noise are added (SNR $=5 \mathrm{~dB}$ and $0 \mathrm{~dB}$ ) medium and high level of noise respectively. The proposed ST is applied on $x_{2}(t)(a=30, b=40$ and $c=150)$ and $x_{3}(t)$ signals and the results are shown in Figs. 7 and 8.

For the four duration transients signal $x_{2}(t)$, the optimized Assou's method and Sejdic's ST still suffer from bad temporal resolution in low frequency (especially for medium level of noise $\mathrm{SNR}=5 \mathrm{~dB})$. For high level of noise $(\mathrm{SNR}=0 \mathrm{~dB})$ it is always possible to distinguish the four transient components (Fig. 6.b and 6.c) but the influence of noise becomes serious. For the crosscomponent signal $x_{3}(t)$, in the presence of high level of noise
(SNR $=0 \mathrm{~dB}$ ) the optimized Assou's method fails to give pertinent results (Fig. 7.c). Its performance does not exceed much more the standard ST. For a medium level of noise (SNR $=5 \mathrm{~dB})$, the optimized Assou's method gives a better resolution for the non-linear component than Sejdic's ST (Fig. 7.b) in high frequency but worth resolution for the linear chirp.

The results given in Fig. 7.d and 8.d illustrate visually the improvement of the proposed methodology comparing to the other versions of ST.

\subsubsection{Estimating the instantaneous frequency}

In order to complete the discussion on the behavior of the proposed ST in the presence of noise, the instantaneous frequency 

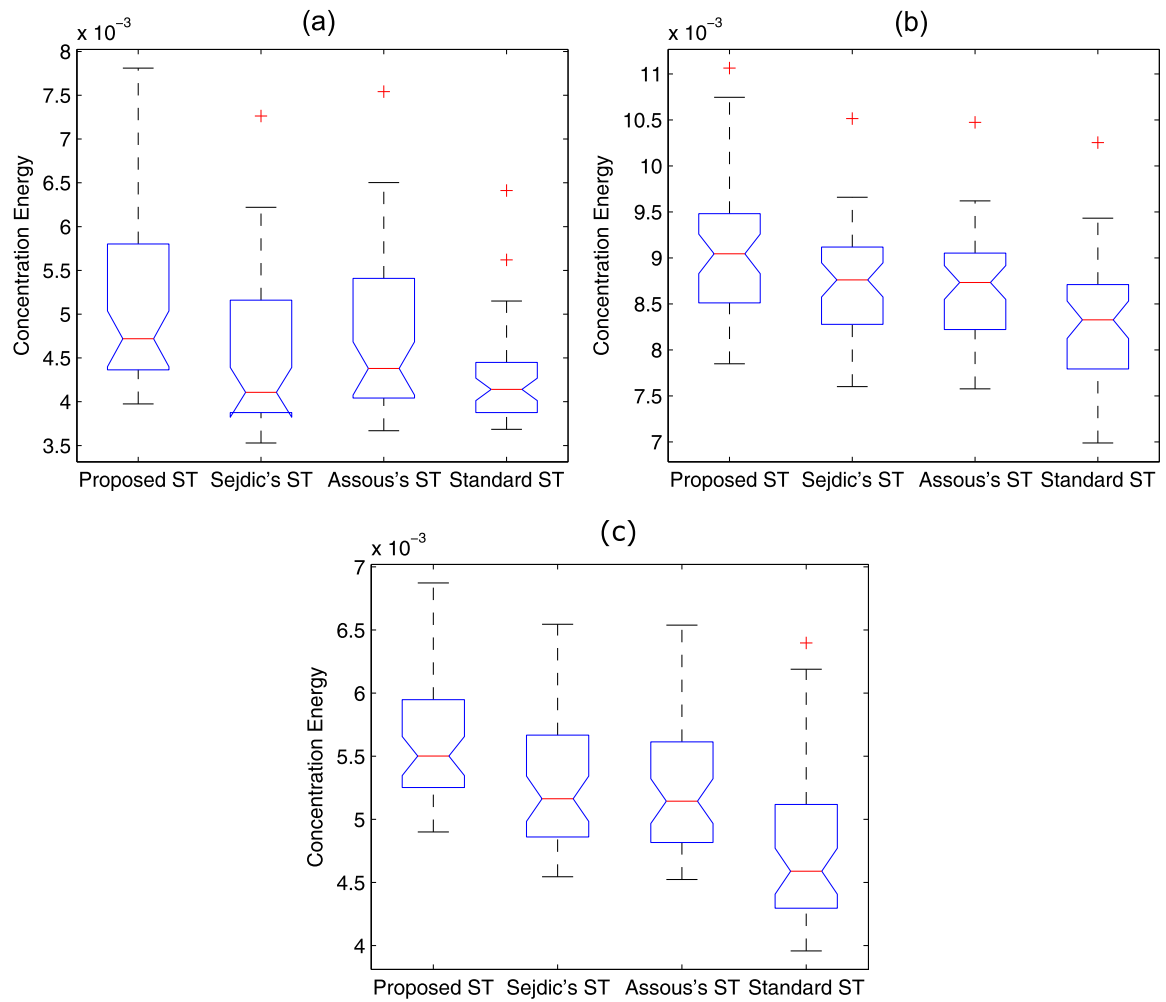

Fig. 6. Boxplot of the concentration energy measures for the different ST methods: (a) $x_{1}(t)$; (b) $x_{2}(t)$; (c) $x_{3}(t)$.

is estimated for seven levels of noise $(0-30 \mathrm{~dB})$ where 1000 realizations are used for each level and the mean square error (MSE) between the analytic equation of the instantaneous frequency (IF) and the obtained IF from time-frequency representation is compared between the different ST methods. A linear chirp $\cos \left(20 \pi t+150 t^{2}\right)$ is used in this test. The estimation of the instantaneous frequency is performed based on the peaks values of the S-matrix and the obtained MSE results are resumed in Fig. 9. The results show clearly the advantageous of the proposed methodology to estimate the instantaneous frequency in the presence of noise comparing the other methods. The proposed method reached the lowest MSE in all cases. The standard ST gives best estimation than Sejdic's ST and optimized Assous's ST in the case of low and middle levels of noise. However, for high level of noise (SNR $=0 \mathrm{~dB}$ ) the MSE of Sejdic's ST and optimized Assous's ST is lower than the standards ST. Fig. 10 illustrates an example of the estimated IF for each method compared with the analytical expression.

The main reason of the high performance of the proposed ST is the flexibility of the proposed window. In the presence of noise the transform is able to adapt to the noisy signal and provide high energy concentration and high performance in the estimation of the IF. The active-set algorithm generate correctly the adequate parameters even if the signal is contaminated with high level of noise.

\subsection{Comparison between different time-frequency representations}

The SPWVD gives a bad time-frequency resolution for the sinusoidal modulated component in the example of $x_{1}(t)(a=150$, $b=20$ and $f=5$ ) (Fig. 11). However, for the linear chirp component the SPWVD gives the better resolution. The proposed ST gives a good resolution for the two components (Fig. 11.c). For the $x_{2}(t)$ example (Fig. 12). However for the signal $x_{3}(t)$ in the multicomponent zone (Fig. 13) the transform still suffers from interference terms and from poor resolution for the non-linear chirp compo-
Table 2

Shows the concentration energy measures (CM) for different Time-Frequency representations applied on synthetic signals.

\begin{tabular}{llll}
\hline CM & STFT & SPWVD & Proposed ST \\
\hline$x_{1}(t)$ & 0.0039 & 0.0050 & 0.0050 \\
$x_{2}(t)$ & 0.0053 & 0.0095 & 0.0079 \\
$x_{3}(t)$ & 0.0039 & 0.0051 & 0.0050 \\
\hline
\end{tabular}

Table 3

Shows the corresponding computational time (in second) of the different ST methods for each synthetic signal (150 frequency voices $* 1000$ samples number for the 3 different signals).

\begin{tabular}{lllll}
\hline Signal & Standard & Sej. ST & Aso. ST & Proposed \\
\hline$x_{1}(t)$ & 0.3 & 1.9 & 0.83 & 2.1 \\
$x_{2}(t)$ & 0.3 & 1.9 & 0.9 & 1.87 \\
$x_{3}(t)$ & 0.3 & 1.9 & 1.1 & 1.78 \\
\hline
\end{tabular}

nent at high frequencies. The proposed S-transform in this paper gives a good compromise overall the time frequency plane. We note here that the concentration energy measure $(\mathrm{CM})$ is a quantitative measure and not a qualitative one. This can explain the higher CM for SPWVD method in the case of signal $x_{3}(t)$ while it's visually clear from Fig. 13 that the quality of the time-frequency representation provided by SPWVD is lower than the proposed ST (Fig. 13.c). The concentration energy results for each method and for one example of each signal are resumed in Table 2.

\subsection{Computational time}

Table 3 shows the computational time (in second) of the proposed ST and other modified ST for each synthetic signal. The proposed method does not acquire a huge computational time. As example, to compute an example of the synthetic signal $x_{2}(t)$ (150 frequency voices $* 1000$ samples number) on MATLAB ${ }^{\circledR}$ and a standard PC configurations the computing time is around $1 \mathrm{sec}-$ 


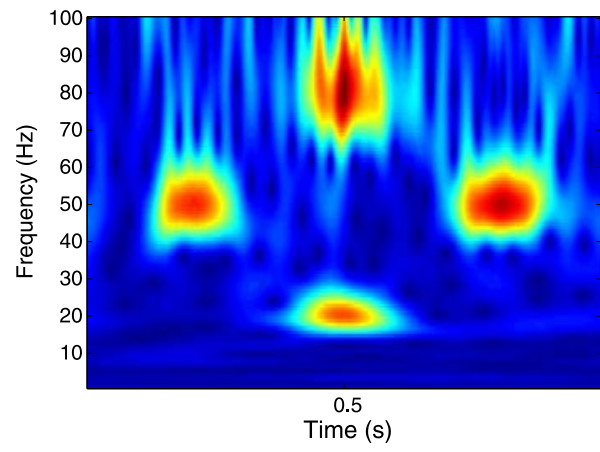

(a)

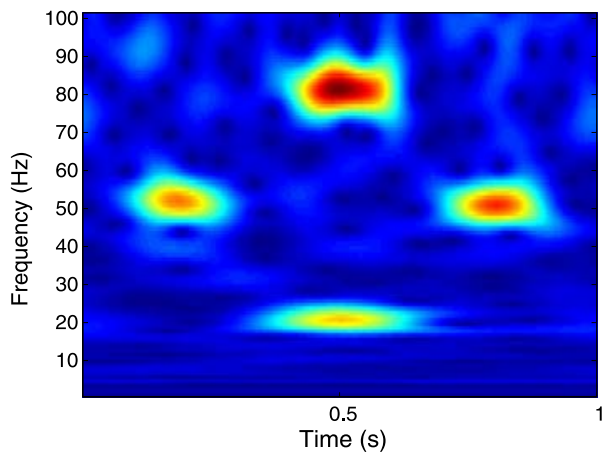

(b)
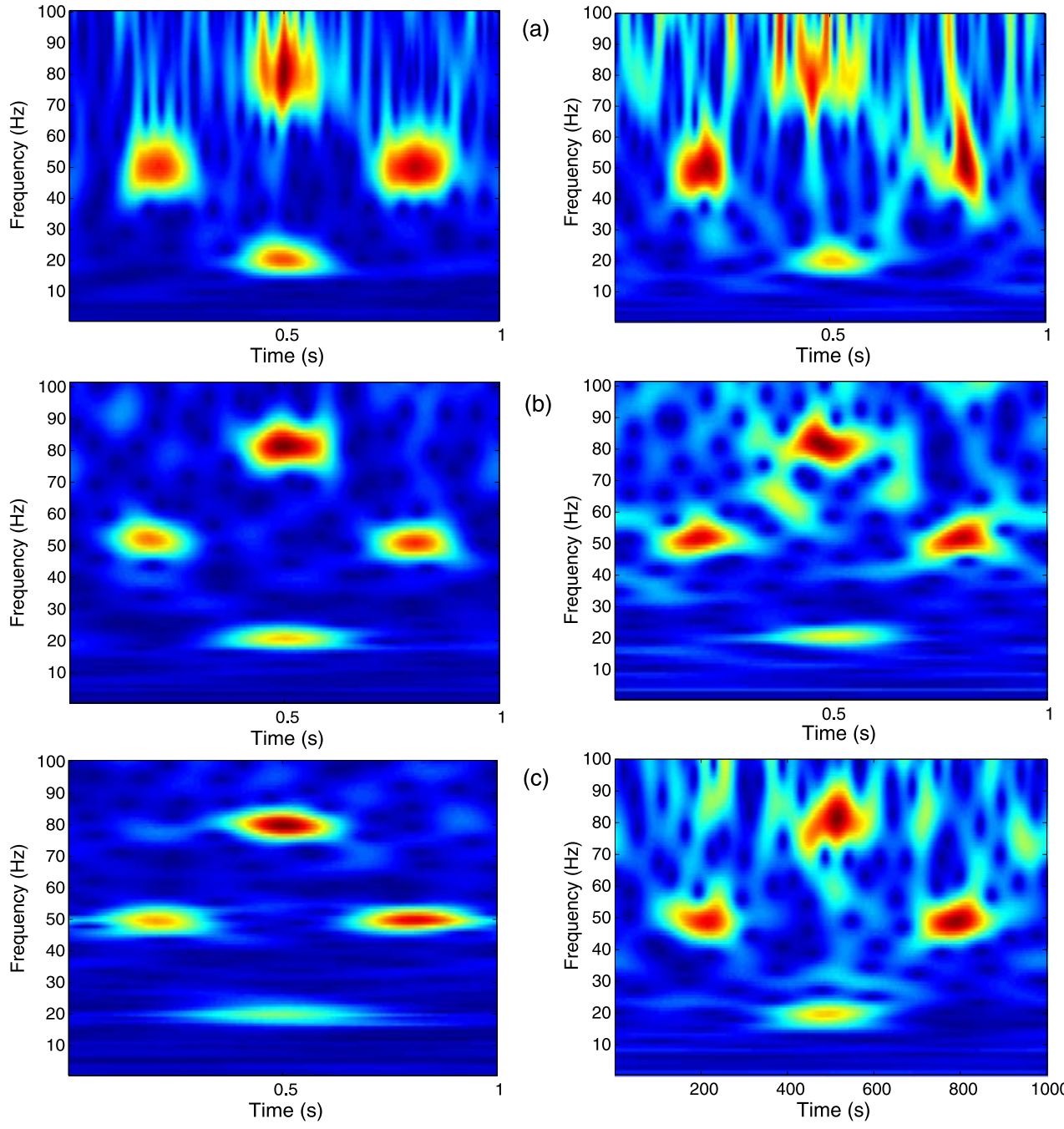

(c)
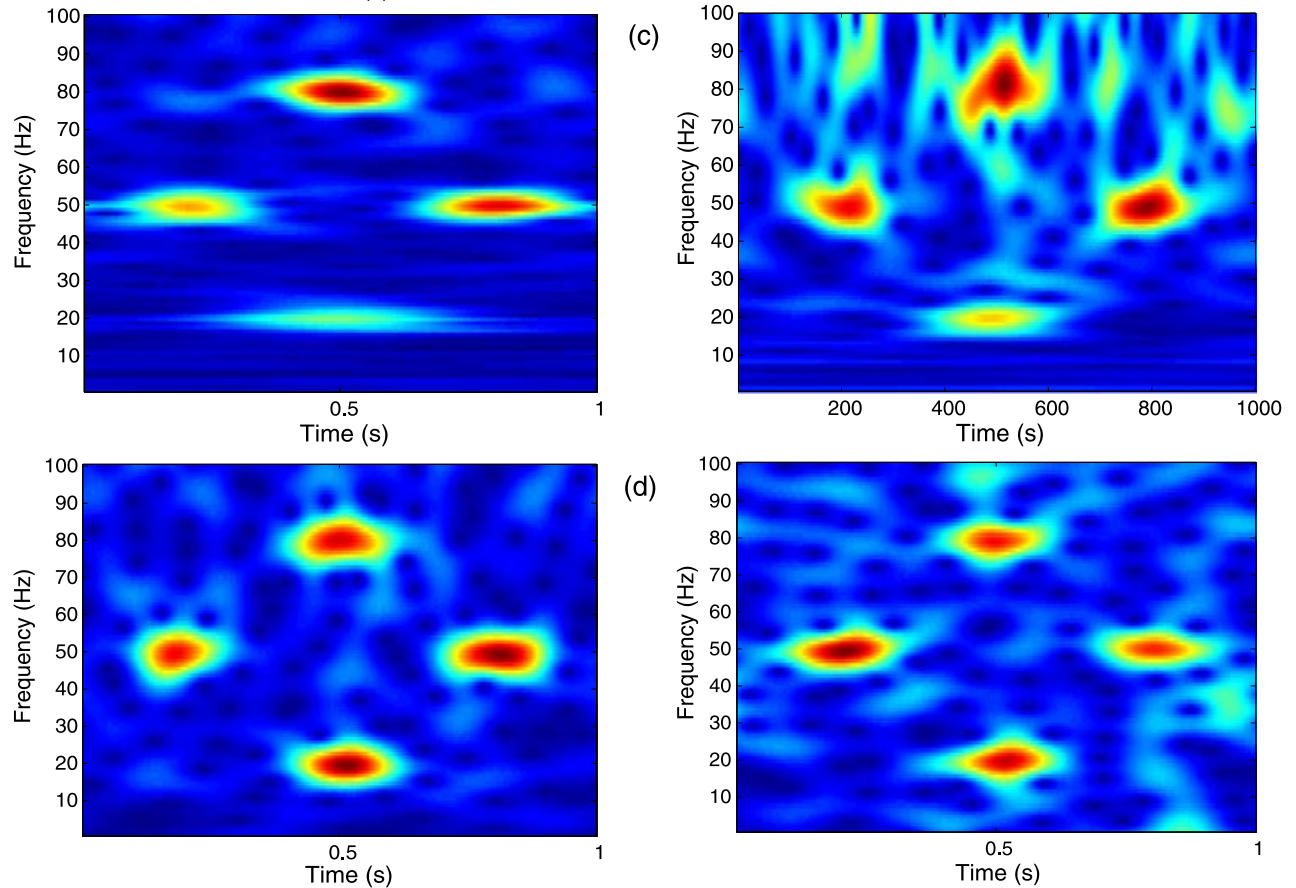

(d)

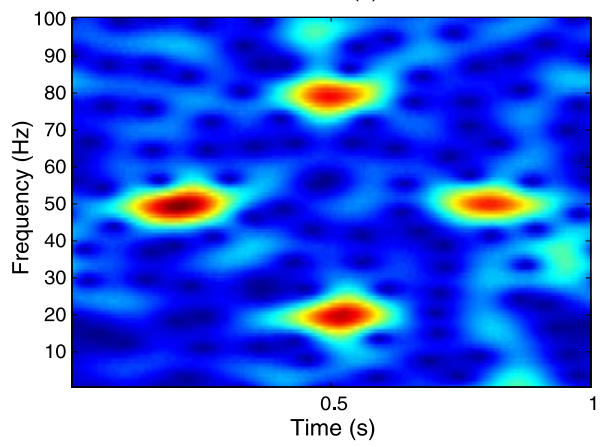

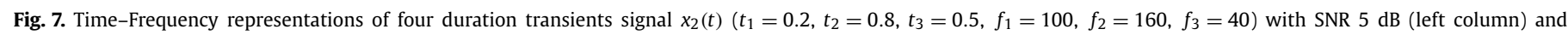
SNR $=0$ dB (right column): (a) Standard ST; (b) Sejdic's ST; (c) Optimized Assous's ST; (d) The proposed ST.

ond. There are no significant differences in the computational complexity between the proposed scheme and the others optimization schemes proposed in the literature. The proposed scheme takes a similar computational time as Sejdic's ST method and little more than the optimized Assous's method. Indeed, for real time applications the computing time can be much more enhanced by implementing the proposed method on DSP or FPGA platforms for example.

\section{Application on real non-stationary signals: heart sounds}

The analysis of the cardiac sounds solely based on the human ear is limited by the experience of the clinician for a reliable di- agnosis of cardiac pathologies and to obtain all the qualitative and quantitative information about cardiac activity. Proposing an objective signal processing methods able to extract relevant information from heart sounds is a great challenge for specialists and auto-diagnosis fields. The electronic stethoscope is capable to register and optimize the quality of the acoustic heart signal, completed by the PhonoCardioGraphic (PCG) presentation of the auscultation signal. The localization of the first and the second heart sounds (S1 and S2), the number of their internal components, their frequential content, etc. can be considered as pertinent information very useful for patricians and for classification systems [6]. The application proposed in this paper consists to detect splits in heart sounds. The split within the S1 and the S2 heart 

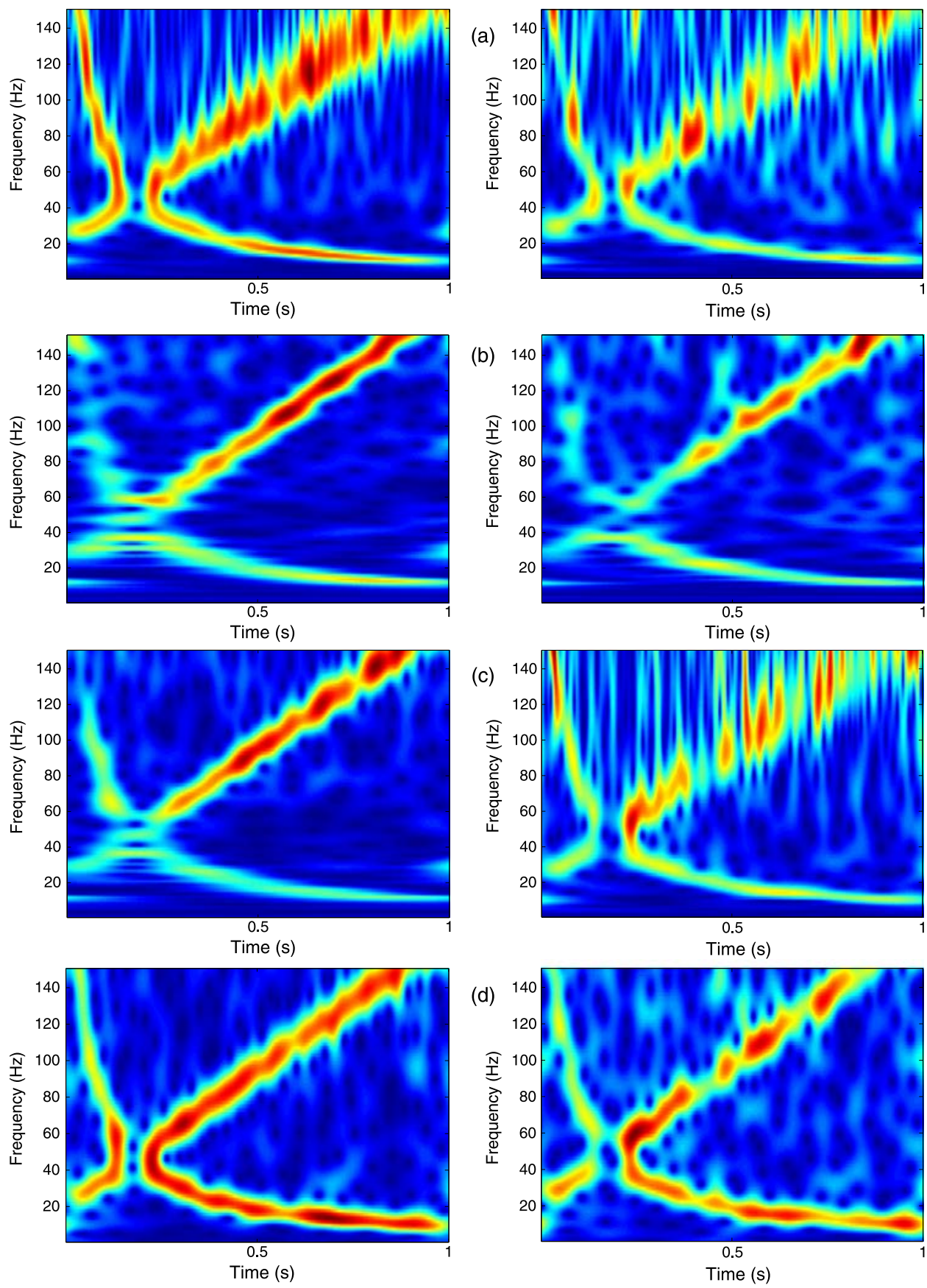

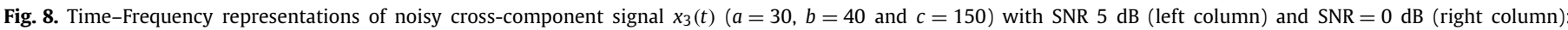
(a) Standard ST; (b) Sejdic's ST; (c) Optimized Assous's ST; (d) The proposed ST.

sounds emerged as an indicator of several valvular diseases [20]. In addition, the PCG signal is a powerful tool for assessing the pulmonary artery pressure. $\mathrm{Xu}$ et al. found out that the pulmonary artery pressure is correlated with the split in S2 [21]. The modified S-transform proposed in this paper is being applied to the PCG signal to detect split and calculate its duration. The optimization of the time-frequency representation of heart sounds can lead to more objective and reliable methods and diagnostics. The proposed algorithm to detect splits in heart sounds can be summarized as follows:

- First, the heart sound is segmented by using the proposed algorithm in [6] to detect the first and the second heart sounds.
- We calculate the optimized S-transform $S_{x}^{m, p, k, r}$ for each segmented sound.

- Then, we calculate the envelope of the segmented sound $x_{i}$ based on the optimized S-transform as follows:

$$
\operatorname{Env}\left(x_{i}\right)=-\int_{-\infty}^{+\infty}\left|S_{x}^{m, p, k, r}(\tau, f)\right|^{2} \log \left(\left|S_{x}^{m, p, k, r}(\tau, f)\right|^{2}\right) d f
$$

- Finally, we apply an algorithm to detect the local extrema of the extracted envelope. Normally, a heart sound with split is supposed to have two local extrema in its extracted envelope. The duration of split is calculated as the distance between the two detected extrema. 
Fig. 14 shows the time-frequency representations of a real heart sound (S1) acquired with a Littmann electronic stethoscope with a clear split. The STFT does not separate the two components due to its poor time-frequency resolution. However, the SPWVD and the proposed ST show a better performances. Fig. 15 shows the envelope calculated by equation (35) and applied on each transform. It favorites the middle amplitudes and attenuate the high and low amplitudes which can help to separate the two components in noisy environment.

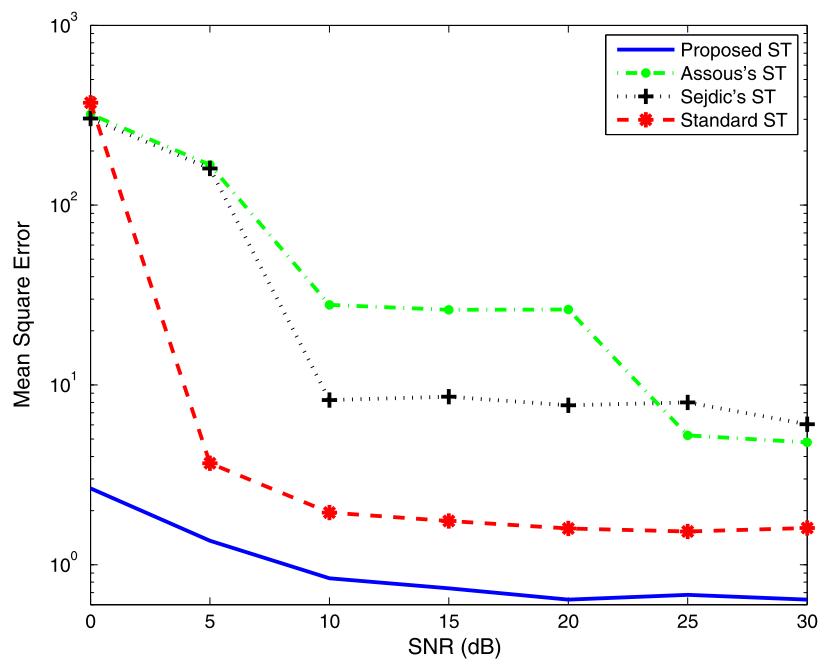

Fig. 9. MSE for the instantaneous frequency for linear chirp with different levels of noise (from 0 to $30 \mathrm{~dB}$ ).

\section{Conclusion}

A new methodology is presented in this paper to enhance the time-frequency resolution of the Stockwell transform. The methodology is based on a new hybrid Gaussian window with several parameters to control the width. For the first time, an optimization process based on active-set method is applied to select properly the window's parameters. This methodology enhances significantly the time-frequency resolution of the Stockwell transform and for the different existing modified S-transform in the literature. These results are confirmed in the presence of Gaussian additive noise and in the estimation of instantaneous frequency from time-frequency plane. Moreover, the proposed method showed a better resolution than some classic time-frequency methods as the STFT and the SPWVD (especially in multicomponent signals with non-linear chirp and sinusoidal modulated components). The performance of the proposed S-transform is confirmed on real nonstationary signals through an example of split detection in heart sounds. The method can be useful for other applications related to non-stationary signals and the optimized parameters $m, p, k$ and $r$ can be used as features for classification schemes.

\section{Annex}

We provide the MATLAB code of the main functions involved in the proposed Stockwell transform
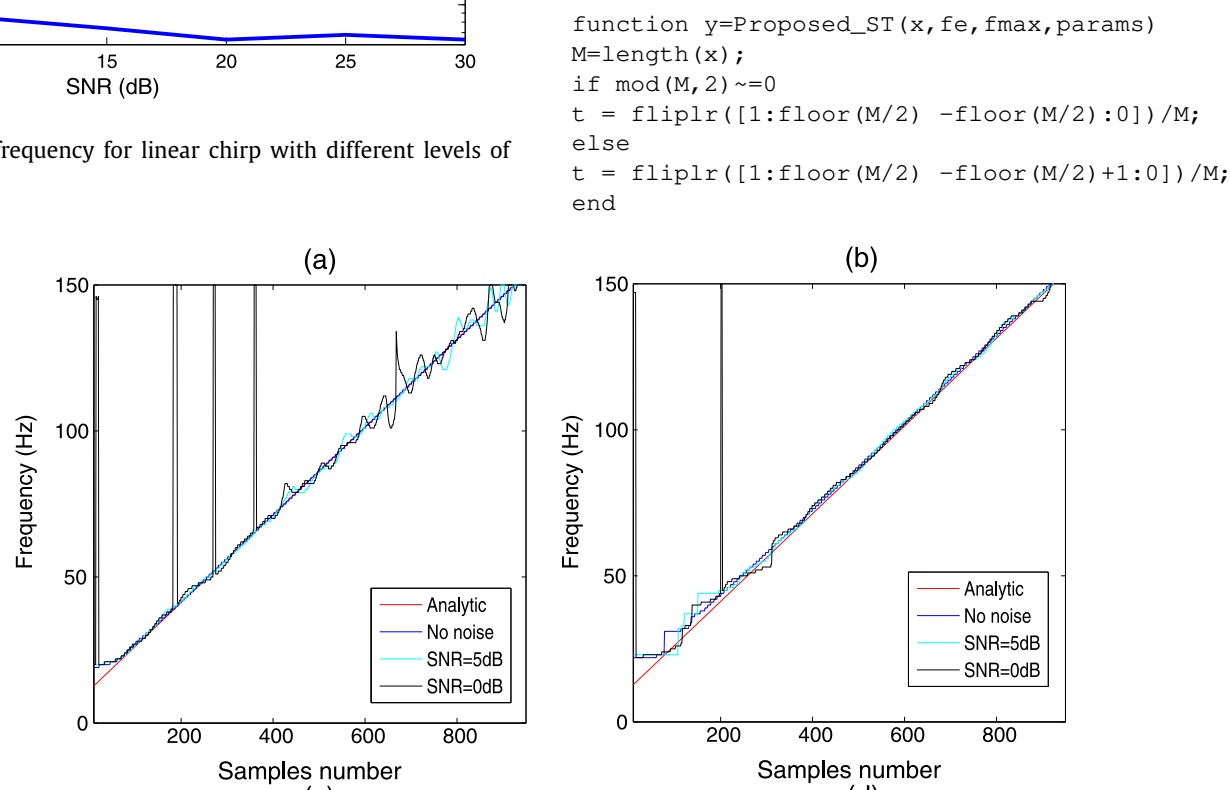

(c)

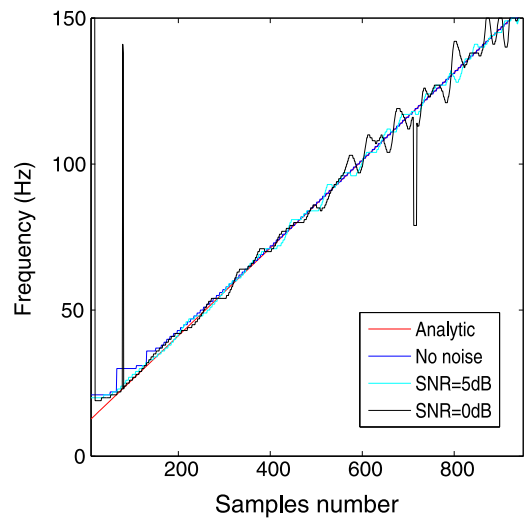

(b)

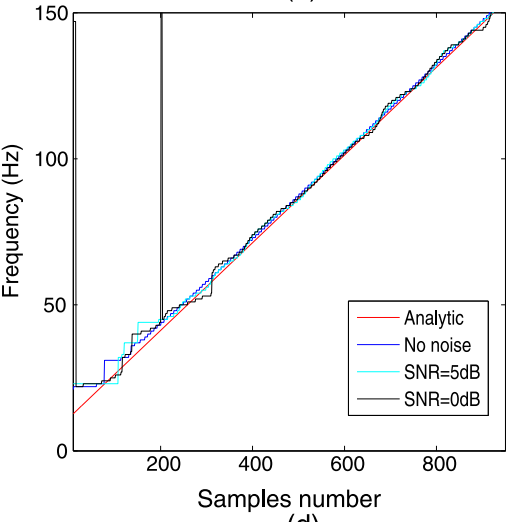

(d)

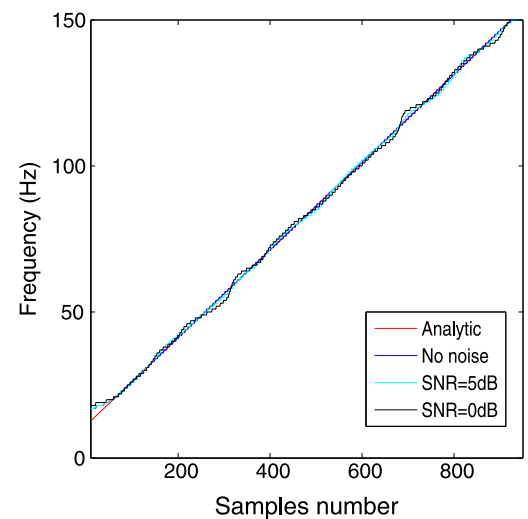

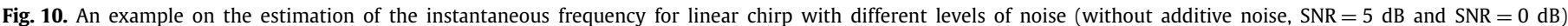
compared with the analytical expression of the IF for: (a) Standard ST; (b) Sejdic's ST; (c) Optimized Assous's ST; (d) The proposed ST. 
(a)

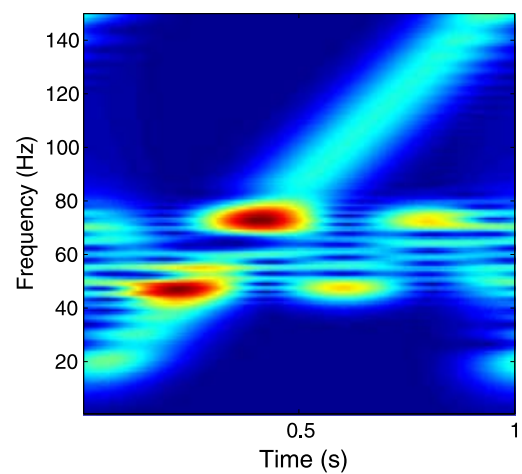

(b)

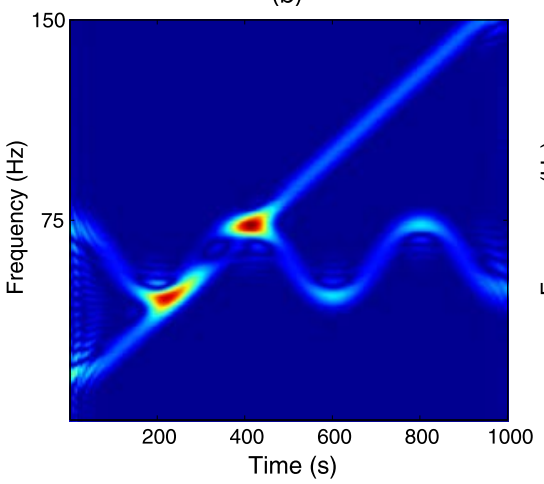

(c)

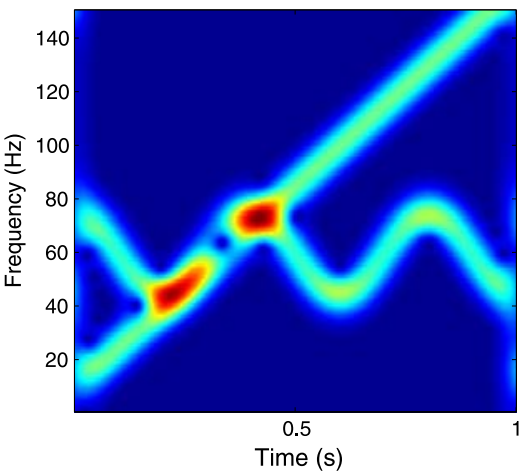

Fig. 11. Time-Frequency representations of signal $x_{1}(t)(a=150, b=20$ and $f=5)$ : (a) STFT; (b) SPWVD; (c) The proposed ST.

(a)

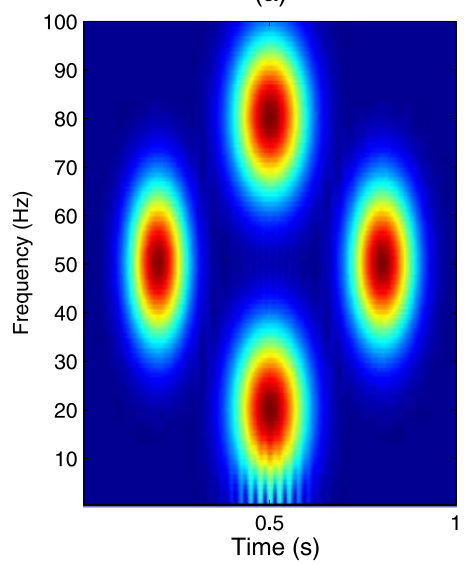

(b)

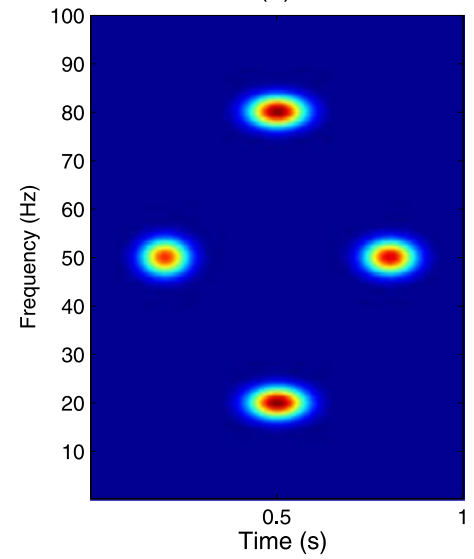

(c)

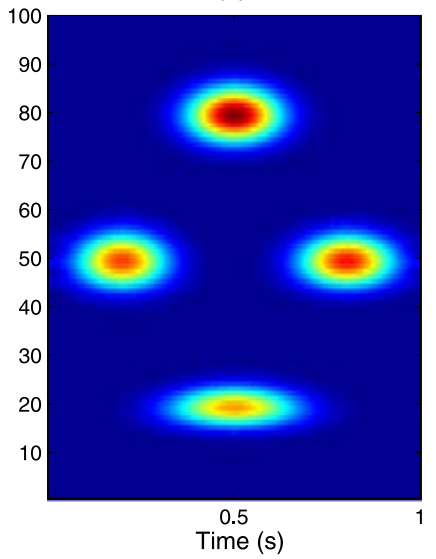

Fig. 12. Time-Frequency representations of four duration transients signal $x_{2}(t)\left(t_{1}=0.2, t_{2}=0.8, t_{3}=0.5, f_{1}=100, f_{2}=160, f_{3}=40\right)$ : (a) STFT; (b) SPWVD; (c) The proposed ST.

(a)

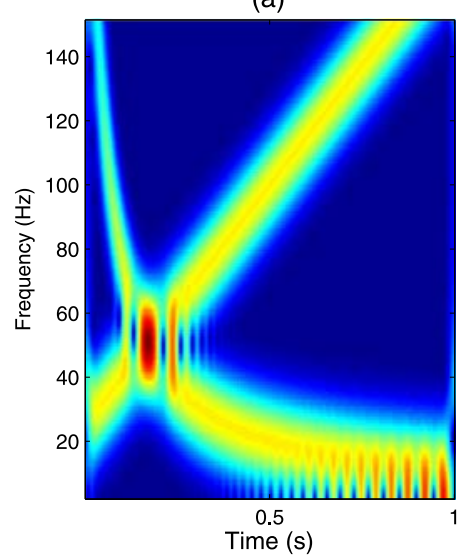

(b)

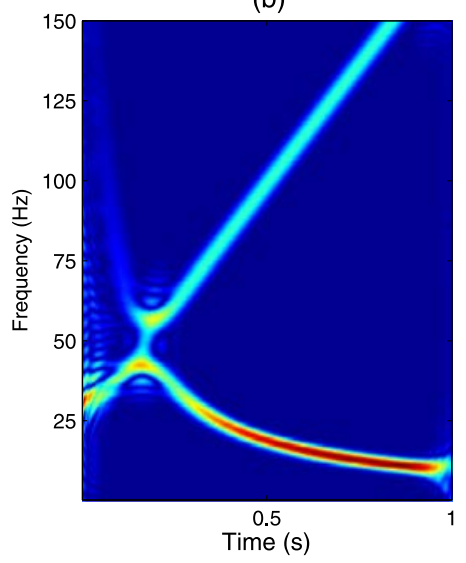

(c)

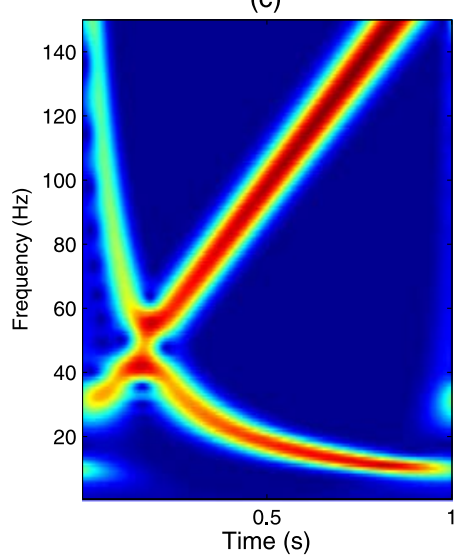

Fig. 13. Time-Frequency representations of cross-component signal $x_{3}(t)(a=30, b=40$ and $c=150)$ : (a) STFT; (b) SPWVD; (c) The proposed ST. 


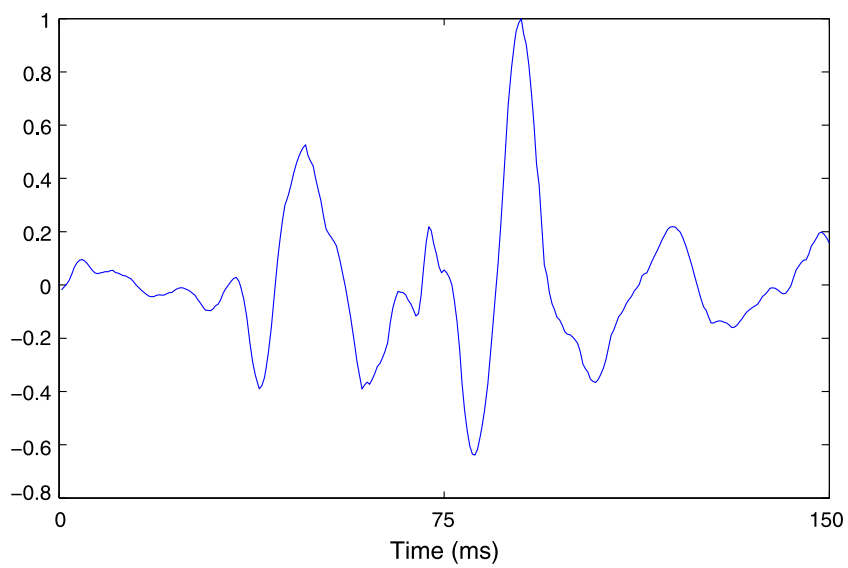

(a)

(b)
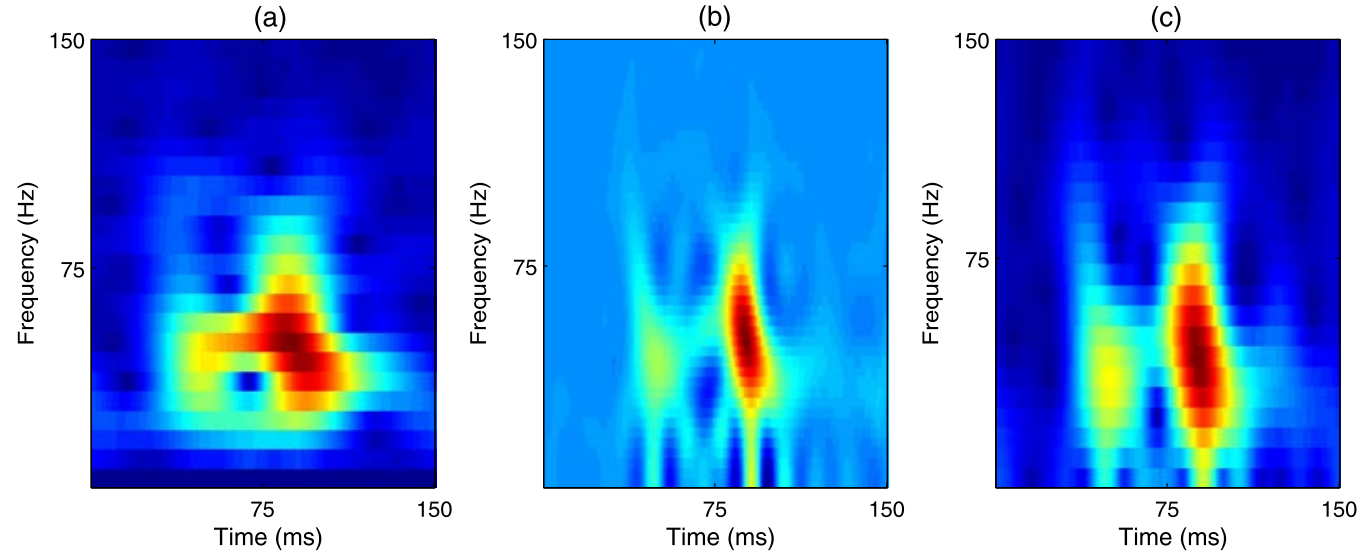

Fig. 14. Temporal real heart sound S1 (top) and its time-frequency representations: (a) The STFT; (b) The SPWVD; (d) The proposed ST.

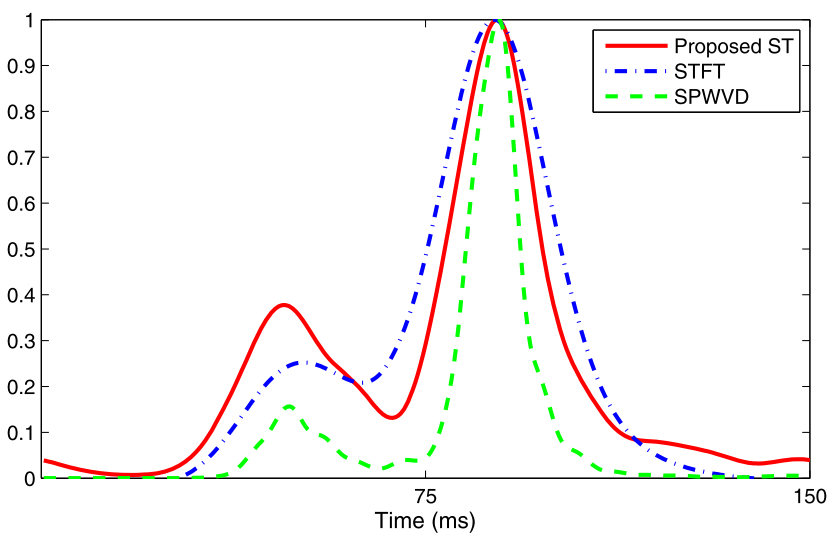

Fig. 15. The envelope given in equation 43 calculated on the example of Fig. 8 for: (solid line) the proposed ST; (dashed dotted line) the STFT; (dashed line) the SPWVD.

-The obtained parameters of the proposed Gaussian window $\mathrm{r}=$ params (1); m=params (2); $\mathrm{p}=$ params (3); $\mathrm{k}=$ params (4); $\mathrm{Fx}=\mathrm{fft}(\mathrm{x}) ; \mathrm{XF}=[\mathrm{Fx} F \mathrm{Fx}]$;

oCalculate the number of steps to compute the S_matrix \%fe: sampling frequency in $\mathrm{Hz}$

ofmax: maximum frequency to calculate the S_transform $\mathrm{Pas}=(\mathrm{fmax} \star \mathrm{M} / \mathrm{fe})$;

otic

for $f=1:$ Pas $\%$ loop to compute the $S$ matrix

oCompute the proposed window

wind $=\left(\left(f^{\wedge} r\right) /\left(\operatorname{sqrt}(2 \star p i) *\left(m *(f)^{\wedge}(p)+k\right)\right)\right) \ldots$

$\star \exp \left(-0.5 *\left(1 /\left(\left(\mathrm{m} *(\mathrm{f})^{\wedge}(\mathrm{p})+\mathrm{k}\right) \cdot \wedge^{\wedge} 2\right)\right) *\left(\mathrm{f}^{\wedge}(2 * \mathrm{r})\right) * t . \wedge 2\right)$;

wind $=$ wind $/($ sum(wind) $)$; onormalization

$\mathrm{W}=\mathrm{fft}(\mathrm{wind})$;

S_matrix $(f,:)=\operatorname{ifft}(X F(f+1: f+M) . * W)$; end

otoc

-Normalize the S matrix

N_S_matrix $=$ S_matrix/sqrt (sum (sum(S_matrix.

*conj (S matrix))) );

○Compute the Concentration Measure

CM_proposed=1/sum (sum (abs (N_S_matrix))) ;

$\mathrm{y}=\mathrm{S} \_$matrix;

\}

function $[r, m, p, k]=o p t i m S T$

optimst is the function that performs the active-set

obased algorithm to generate the optimized parameters

ObjectiveFunction $=$ e (v) Fitness_Function (v, x, Fs, fmax);

nvars $=4 ; \quad \frac{\circ}{0}$ Number of variables

$\mathrm{LB}=\left[\begin{array}{llll}0 & 0 & 0 & 0\end{array}\right] ; \quad$ Lower bound

$\mathrm{UB}=\left[\begin{array}{llll}3 & 3 & 3 & 3\end{array}\right] ; \quad \%$ Upper bound

$\mathrm{x} 0=\left[\begin{array}{llll}1 & 1 & 1 & 1\end{array}\right]$; \%start point

ConstraintFunction = esimple_constraint

options = optimoptions (@fmincon, 'Algorithm', 'Active-set',

'Display',' off');

$[\mathrm{x}, \mathrm{fval}]=$ fmincon $(\mathrm{Object}$ iveFunction, $\mathrm{x} 0,[],[],[], \ldots$

[] , LB, UB, ConstraintFunction, options)

$\mathrm{r}=\mathrm{x}(1) ; \mathrm{m}=\mathrm{x}(2) ; \mathrm{p}=\mathrm{x}(3) ; \mathrm{k}=\mathrm{x}(4)$;

$\mathrm{y}=[\mathrm{r}, \mathrm{m}, \mathrm{p}, \mathrm{k}]$;

end

function $\mathrm{y}=$ Fitness Function $(\mathrm{v}, \mathrm{x}, \mathrm{fe}$, fmax)

$\mathrm{A}=\mathrm{v}(1) ; \mathrm{m}=\mathrm{v}(2) ; \mathrm{p}=\mathrm{v}(3) ; \mathrm{k}=\mathrm{v}(4)$;

$\mathrm{X} 1=\mathrm{ST}$ Proposed $(\mathrm{x}, \mathrm{fe}, 1, \mathrm{fmax},[\mathrm{A}, \mathrm{m}, \mathrm{p}, \mathrm{k}])$;

$\mathrm{X} 1 \mathrm{p}=\mathrm{X} 1 / \operatorname{sqrt}(\operatorname{sum}(\operatorname{sum}(\mathrm{X} 1 . \star \operatorname{con} j(\mathrm{X} 1)))) ;$

$\mathrm{CM}=1 / \operatorname{sum}(\operatorname{sum}(\mathrm{abs}(\mathrm{X} 1 \mathrm{p})))$;

$\mathrm{y}=-\mathrm{CM}$;

end

function $[\mathrm{c}, \mathrm{ceq}]=$ simple_constraint $(\mathrm{x})$

fmax $=100$; Ts=1/1000; 
$\mathrm{C}=\left[10 * \mathrm{Ts} \star(\operatorname{fmax})^{\wedge} \mathrm{x}(1)-\mathrm{x}(4)-\mathrm{x}(2) ;\right.$

$\mathrm{x}(2) \star(\operatorname{fmax}) \wedge x(3)-0.1+\mathrm{x}(4)]$;

ceq $=[] ;$

\section{References}

[1] A. Moukadem, D. Ould Abdeslam, A. Dieterlen, Time-Frequency Domain for Segmentation and Classification of Non-Stationary Signals, Wiley-ISTE, ISBN 978-1-84821-613-6, 2014

[2] R.G. Stockwell, L. Mansinha, R. Lowe, Localization of the complex spectrum: the S transform, IEEE Trans. Signal Process. 44 (4) (1996) 998-1001.

[3] L. Cheng, G. William, Z. Hongmei, Estimation of time-varying coherence and its application in understanding brain functional connectivity, EURASIP J. Adv. Signal Process. 2010 (2010).

[4] E. Sejdic, J. Jiang, Selective regional correlation for pattern recognition, IEEE Trans. Syst. Man Cybern., Part A, Syst. Hum. 37 (1) (2007) 82-93.

[5] G. Livanos, N. Ranganathan, J. Jiang, Heart sound analysis using the S transform, in: Computers in Cardiology 2000, 2000, pp. 587-590.

[6] A. Moukadem, A. Dieterlen, N. Hueber, C. Brandt, A robust heart sounds segmentation module based on S-transform, Biomed. Signal Process. Control 8 (3) (2013) 273-281.

[7] M. Jaya Bharata Reddy, R.K. Raghupathy, K. Venkatesh, D. Mohanta, Power quality analysis using discrete orthogonal s-transform (dost), Digit. Signal Process. 23 (2) (2013) 616-626

[8] C.R. Pinnegar, H. Khosravani, P. Federico, Time-frequency phase analysis of ictal EEG recordings with the S-transform, IEEE Trans. Biomed. Eng. 56 (11) (2009) 2583-2593.

[9] E. Sejdic, I. Djurovic, J. Jiang, A window width optimized S-transform, EURASIP J. Adv. Signal Process. 2008 (2008) 59.

[10] P. McFadden, J. Cook, L. Forster, Decomposition of gear vibration signals by the generalised S transform, Mech. Syst. Signal Process. 13 (5) (1999) 691-707.

[11] C.R. Pinnegar, L. Mansinha, The S-transform with windows of arbitrary and varying shape, Geophysics 68 (1) (2003) 381-385.

[12] C.R. Pinnegar, L. Mansinha, The bi-Gaussian S-transform, SIAM J. Sci. Comput 24 (5) (2003) 1678-1692.

[13] I. Djurović, E. Sejdić, J. Jiang, Frequency-based window width optimization for S-transform, AEÜ, Int. J. Electron. Commun. 62 (4) (2008) 245-250.

[14] S. Assous, B. Boashash, Evaluation of the modified S-transform for timefrequency synchrony analysis and source localisation, EURASIP J. Adv. Signal Process. 2012 (1) (2012) 1-18.

[15] K. Gröchenig, Foundations of Time-Frequency Analysis, Springer, 2001.

[16] A. Moukadem, Z. Bouguila, D.O. Abdeslam, A. Dieterlen, Stockwell transform optimization applied on the detection of split in heart sounds, in: 22nd European Signal Processing Conference, 2014.

[17] L. Mansinha, R. Stockwell, R. Lowe, M. Eramian, R. Schincariol, Local S-spectrum analysis of 1-D and 2-D data, Phys. Earth Planet. Inter. 103 (3) (1997) 329-336.

[18] N.V. George, S.S. Sahu, L. Mansinha, K. Tiampo, G. Panda, Time localised band filtering using modified S-transform, in: Proceedings on the IEEE International Conference on Signal Processing Systemes (ICSPS), Singapore, 2009, pp. 42-46.
[19] L. Stanković, A measure of some time-frequency distributions concentration, Signal Process. 81 (3) (2001) 621-631.

[20] A. Djebbari, F. Bereksi-Reguig, Detection of the valvular split within the second heart sound using the reassigned smoothed pseudo Wigner-Ville distribution, Biomed. Eng. Online 12 (1) (2013) 37.

[21] K.-S. Tang, K. Man, S. Kwong, Q. He, Genetic algorithms and their applications, IEEE Signal Process. Mag. 13 (6) (1996) 22-37. 